ISSN: 0210-1696

DOI: http://dx.doi.org/10.14201/scero20154634771

\title{
LA TRANSFORMACIÓN DE LOS SERVICIOS HACIA LA CALIDAD DE VIDA. UNA INICIATIVA DE INNOVACIÓN SOCIAL DE FEAPS
}

\section{The Transformation of Services towards the Quality of Life. An Initiative of Social Innovation from FEAPS}

Javier TAMARIT CUADRADO Confederación FEAPS. Madrid. Avda. del General Perón, 32, 1. . 28020 Madrid calidad@feaps.org

Recepción: 26 de noviembre de 2014

Fecha de aceptación definitiva: 15 de junio de 2015

Biblid. [0210-1696 (2015) vol. 46 (3), n. ${ }^{\circ} 255$, julio-septiembre; 47-71]

RESUMEN: Este artículo presenta la base conceptual y estratégica del proyecto que está desarrollando FEAPS, movimiento asociativo español en favor de las personas con discapacidad intelectual o del desarrollo y sus familias, para avanzar en la transformación de sus centros y servicios hacia servicios centrados en la persona, orientados a su calidad de vida y a la plena ciudadanía en comunidades más inclusivas. La transformación es un proceso de ámbito internacional en el campo de la discapacidad intelectual o del desarrollo y cuenta con una base importante de conocimiento, pero además es un proceso derivado de la convención de los derechos de las personas con discapacidad y que por tanto tiene impacto en las políticas públicas. En estos momentos en FEAPS se están desarrollando experiencias de transformación en el ámbito de la atención temprana (38 centros de 10 comunidades diferentes), la educación (38 colegios de 10 comunidades diferentes), centros ocupacionales (una experiencia inicial en Madrid con 9 centros ocupacionales), apoyo a mayores con discapacidad intelectual o del desarrollo (con 19 entidades de 4 comunidades autónomas). Tras describir estas experiencias se muestra el proceso general que se está siguiendo y algunas de las claves de aprendizaje derivadas de este proyecto. 
PALABRAS Clave: transformación organizacional; servicios centrados en la persona; discapacidad intelectual o del desarrollo; calidad de vida; experiencias de transformación de servicios; innovación social.

Aвstract: This paper presents the conceptual and strategic basis of the project that is being developed by FEAPS, the social movement of Spanish associations for people with intellectual and developmental disabilities and their families, to advance on the transformation of their centres and services towards person-centred services, oriented to the quality of life and full citizenship in more inclusive communities. Transformation is a process of international scope in the field of intellectual and developmental disabilities and has a strong knowledge base, but it is also a process derived from the Convention on the Rights of Persons with Disabilities and therefore it impacts in public policy. Currently, FEAPS is developing transformative experiences in the field of early intervention (38 centres in 10 different regions), education (38 schools in 10 different regions), work-sheltered centres (an initial experience in Madrid with nine work-sheltered centres), and services of support for older people with intellectual or developmental disabilities (with 19 entities in 4 regions). After describing these experiences the overall process that is being followed and some key learning derived from this project are shown.

KEY WORDS: organisational transformation; person-centred services; intellectual or developmental disabilities; quality of life; experiences on services transformation; social innovation.

\section{Introducción. El marco de la transformación organizacional}

4

N LOS POSICIONAMIENTOS INTERNACIONALES tanto de carácter científico y profesional como de las propias organizaciones que representan a las personas con discapacidad intelectual o del desarrollo, se clama desde hace tiempo por la transformación de los modelos y servicios que históricamente se han venido ofreciendo, basados en la asistencia institucional, generando la urgencia del cambio hacia otros servicios de apoyo orientados hacia su calidad de vida (Bradley, 1994; Hickey, 2012; Morgan, 2010; Schalock et al., 2010; Schalock, Gardner y Bradley, 2007; Schalock y Verdugo, 2003, 2013a, 2013b), con la necesidad de transformar también los roles profesionales (Schalock, 2015) y en un marco de derechos y de inclusión social (Boletín Oficial del Estado, 2008). Como se observa en la Tabla 1 (Bradley, 1994) esta demanda de transformación de los servicios es en buena parte fruto del propio devenir cultural e histórico, fruto en definitiva de una historia compartida de reivindicación social y de avance en el conocimiento científico, que nos lleva a intentar lograr de forma permanente las mejores prácticas organizacionales y profesionales (Schalock, 2015). 


\begin{tabular}{|l|l|l|l|}
\hline \multicolumn{2}{|c|}{ TABLA 1. Fases históricas en el desarrollo de los servicios. Adaptado de Bradley, 1994 } \\
\hline Fases en los servicios & \multicolumn{1}{|c|}{ Institucional } & \multicolumn{1}{c|}{ De integración } & \multicolumn{1}{c|}{$\begin{array}{c}\text { Inclusión en la } \\
\text { comunidad }\end{array}$} \\
\hline ¿Quién lo recibe? & Enfermo & Minusválido & Ciudadano \\
\hline ¿Qué se planifica? & Los cuidados & Las habilidades & El futuro \\
\hline $\begin{array}{l}\text { ¿Quién toma las } \\
\text { decisiones? }\end{array}$ & El especialista & El equipo & $\begin{array}{l}\text { La persona con su } \\
\text { círculo de apoyos }\end{array}$ \\
\hline $\begin{array}{l}\text { ¿Qué pretenden } \\
\text { lograr? }\end{array}$ & Cuidar y curar & Enseñar & Vivir \\
\hline $\begin{array}{l}\text { ¿Qué determina la } \\
\text { calidad? }\end{array}$ & $\begin{array}{l}\text { La práctica } \\
\text { profesional y el nivel } \\
\text { de cuidados }\end{array}$ & $\begin{array}{l}\text { La realización } \\
\text { de programas y } \\
\text { objetivos }\end{array}$ & $\begin{array}{l}\text { La calidad de vida de } \\
\text { la persona }\end{array}$ \\
\hline
\end{tabular}

En gran medida, la transformación de las organizaciones y servicios de apoyo a personas con discapacidades del desarrollo, e incluso de otras condiciones de exclusión social y vulnerabilidad social, implica sustituir un modelo mental y organizativo que se centra en el sistema y la gestión más que en las personas para las que existe ese servicio (González, Ortega, Martínez y Tamarit, 2014). Mount (Mount, 2000; Amado y McBride, 2001) diferenciaba claramente (Tabla 2) un Servicio Centrado en el Sistema, en la organización, frente a un Servicio Centrado en la Persona.

\begin{tabular}{|l|l|}
\hline \multicolumn{2}{|c|}{$\begin{array}{c}\text { Tabla 2. Diferencias entre un Servicio Centrado en el Sistema y un Servicio Centrado } \\
\text { en la Persona (Mount, 2000; Amado y McBride, 2001) }\end{array}$} \\
\hline Servicio Centrado en el Sistema & Servicio Centrado en la Persona \\
\hline Planifica la vida de los programas & Esboza un estilo de vida deseable \\
\hline $\begin{array}{l}\text { Ofrece un número limitado de opciones de } \\
\text { programa generalmente segregados }\end{array}$ & $\begin{array}{l}\text { Diseña un número ilimitado de experiencias } \\
\text { deseables }\end{array}$ \\
\hline $\begin{array}{l}\text { Basa las opciones en estereotipos acerca de } \\
\text { las personas con discapacidad }\end{array}$ & $\begin{array}{l}\text { Encuentra nuevas posibilidades para cada } \\
\text { persona }\end{array}$ \\
\hline $\begin{array}{l}\text { Se centra en rellenar camas, rellenar } \\
\text { formularios... }\end{array}$ & Se centra en calidad de vida \\
\hline $\begin{array}{l}\text { Da gran importancia a la tecnología y a } \\
\text { estrategias clínicas }\end{array}$ & $\begin{array}{l}\text { Hace hincapié en los sueños, deseos y } \\
\text { experiencias significativas }\end{array}$ \\
\hline $\begin{array}{l}\text { Se organiza para complacer a los promotores } \\
\text { inspectores... }\end{array}$ & Se organiza para responder a las personas \\
\hline
\end{tabular}

La transformación organizacional es un fenómeno ampliamente estudiado e investigado (Martínez-Tur y Peñarroja, 2012). Se habla de transformación cuando es preciso un cambio cultural de la organización, en nuestro caso este cambio cultural es el cambio de paradigma que lleva de centrarnos en la discapacidad a centrarnos en la persona: "La característica distintiva de la transformación es el cambio cultural de la organización” (Martínez-Tur, Peiró, Moliner y Potocnik, 2010: 27). Y para una 
eficaz transformación se requiere, al menos, una 'masa crítica' de compromiso de quienes forman la organización y un liderazgo que aliente y apoye la transformación, promoviendo equipos transversales para liderar el plan de transformación (equipos en los que estén también, en nuestro ámbito, personas con discapacidad, familiares, además de profesionales o voluntarios), dándoles poder para actuar. Martínez-Tur y sus colaboradores (2010) señalan estas claves que facilitan que se produzca una transformación:

- Permeabilidad: la característica de una organización de permitirse ser influida y modificada por los saberes, experiencias y prácticas fuera de ella. En FEAPS esta es una de las razones de la realización de ediciones bienales de buenas prácticas (Tamarit, 2012).

- Compromiso de valor competitivo o reformador de la cultura existente: la existencia de una masa crítica de pensamiento transformador de la realidad actual es clave.

- Organizaciones democráticas. Esta clave es compartida por las propuestas desarrolladas en la última edición de la AAIDD (American Association on Intellectual and Developmental Disabilities) (Schalock et al., 2010).

- Empoderamiento de las personas, poder para actuar. También, en nuestro caso, es importante el empoderamiento de las personas con discapacidad intelectual o del desarrollo (Tamarit y Espejo, 2013).

Walker (2012) analiza las estrategias comunes a la experiencia del viaje de transformación de cuatro organizaciones proveedoras de servicios a personas con discapacidad intelectual y encuentra en ese proceso cambios culturales, cambios de pensamiento, impacto muy positivo en calidad de vida, transformación mediante comunidades de práctica. Su principal mensaje es que toda organización puede hacer alguna transformación. Las estrategias comunes que encuentra en las experiencias exitosas son (Walker, 2012):

1. Generación de compromiso con la transformación partiendo de una misión y valores comunes, y a través de conversaciones en profundidad, con participación, compartiendo su experiencia con otros y exponiendo sus propuestas con apertura, desarrollando una planificación organizacional orientada por valores y por la misión.

2. Promover el cambio individual, vinculado a una auténtica planificación centrada en la persona, con una profunda colaboración con las personas con discapacidad intelectual y alejándose de programas preconcebidos, tradicionales, y de ideas preconcebidas sobre lo que es posible o sobre lo que está disponible, ya sean estas ideas de los proveedores de los servicios, de las familias o de las propias personas con discapacidad.

3. No es cuestión simplemente de contextos más reducidos, se trata de cambiar el poder y el control, compartiéndolo realmente con las personas que 
reciben el servicio. Ese poder puede venir, entre otros modos, de la mano de presupuestos individualizados o, por ejemplo, de la participación de las personas con discapacidad intelectual en los procesos de selección y contratación de sus personas de apoyo.

4. Usar relaciones y apoyos de la comunidad. La incorporación a los procesos de prestación individualizada de apoyos de personas de la comunidad puede tomar varias formas, por ejemplo, la incorporación de vecinos en los planes de apoyo, bien sea mediante pago económico o sin él.

5. Dejar de estar en contextos basados en recursos e instalaciones. Esto supone tomar postura claramente en relación con el cese de servicios institucionalizados, bien en un corto plazo (por ejemplo, en el plazo de un año) o bien en un plazo de varios años. La experiencia de los participantes en este estudio era que el cese de los recursos institucionalizados lo consideraban el paso más fácil, lo más complejo era el después, el reto de ayudar realmente después a la calidad de vida de la persona.

6. Más allá de la formación de equipos. Se requieren equipos con vinculación positiva con su trabajo, comprometidos, formados en valores y en trabajo en equipo, alimentando la comunicación y la confianza y propiciando su participación plena en los procesos de transformación.

Como conclusiones de este estudio se indican las siguientes (Walker, 2012): no hay un único modo para la transformación organizacional, es un camino complejo y único para cada organización; la transformación es un proceso permanente de aprendizaje; formar comunidades de práctica puede facilitar la transformación; el contexto político puede suponer retos importantes en el proceso de transformación si suponen una burocracia excesiva, con procedimientos engorrosos con una mirada asistencialista; la transformación puede suponer un impacto muy positivo en la calidad de vida de las personas con discapacidad; $y$, finalmente, toda organización puede hacer algún cambio.

Otra propuesta para afrontar y avanzar hacia soluciones efectivas ante fenómenos o problemas complejos y extensos en la sociedad, como es el de la transformación de servicios para las personas con discapacidad intelectual o del desarrollo hacia servicios centrados en la persona, es el modelo de impacto colectivo (Kania y Kramer, 2011). Este modelo implica el compromiso firme de un grupo numeroso de relevantes actores, vinculados a sectores variados, que cuentan con un plan compartido y alineado, orientado a resolver un complejo problema social concreto. Las condiciones para un impacto colectivo eficaz son: contar con una agenda común, un modelo común; tener un sistema de medición compartida; realizar actividades que se refuerzan mutuamente y que van en línea con la agenda común; mantener una comunicación constante entre los participantes; y contar con el respaldo de una organización columna vertebral, eje central, del proyecto (Figura 1). 
Figura 1. Las cinco condiciones del impacto colectivo (tomado de Kania y Kramer, 2011)

\section{Las cinco condiciones del impacto colectivo}

\begin{tabular}{l|l} 
Agenda común & $\begin{array}{l}\text { Todos los participantes tienen una visión compartida para el cambio, } \\
\text { incluida una comprensión común del problema y un enfoque conjunto } \\
\text { para resolverlo a través de acciones acordadas. }\end{array}$ \\
\hline Medición compartida & $\begin{array}{l}\text { La sistemática recopilación de datos y medición de los resultados } \\
\text { entre todos los participantes garantiza que los esfuerzos permanezcan } \\
\text { alineados y que los participantes asuman su responsabilidad. }\end{array}$ \\
\hline $\begin{array}{l}\text { Actividades que se } \\
\text { refuerzan mutuamente }\end{array}$ & $\begin{array}{l}\text { Las actividades de los participantes deben diferenciarse y, a su vez, estar } \\
\text { coordinadas a través de un plan de acción que se refuerza mutuamente. }\end{array}$ \\
\hline Comunicación constante & $\begin{array}{l}\text { La comunicación constante y abierta es necesaria entre los numerosos } \\
\text { participantes para desarrollar confianza, garantizar objetivos mutuos y } \\
\text { crear una motivación común. }\end{array}$ \\
\hline Respaldo de un eje central & $\begin{array}{l}\text { La creación y gestión del impacto colectivo exige una organización } \\
\text { aparte con personal y un conjunto muy específico de habilidades } \\
\text { para actuar como el eje central de toda la iniciativa y coordinar a las } \\
\text { organizaciones y agencias participantes. }\end{array}$ \\
\hline
\end{tabular}

En esta propuesta de modelo, que es clave en la orientación de los procesos de transformación que lleva a cabo FEAPS y que más adelante se muestran, no se busca exclusivamente el éxito individual de una acción aislada sino el impacto colectivo de transformación, de muchas acciones que hacen en red este proceso.

Schalock y Verdugo (2013b) hablan de un marco para la transformación organizacional que contempla las siguientes estrategias: la persona en el centro (resultados personales y apoyos individualizados); organizaciones más ligeras (alineamiento, equipos de alto rendimiento); mejora continua de la calidad (construir capacidad, enfoque de cuadro de mando); sistemas de información (perspectivas basadas en la ejecución, indicadores basados en la evidencia), y liderazgo participativo (pensamiento expandido, sinergia).

Para afrontar un proceso de transformación desde la institucionalización hacia servicios incluidos en la comunidad se desarrollan algunas experiencias (Van Loon, 2006) o propuestas, como la desarrollada por Parker (2011) mediante una lista de chequeo para avanzar en el cumplimiento por parte de los gobiernos del artículo 19 de la Convención sobre los derechos de las personas con discapacidad (ver Tabla 3). 
TABLA 3. Decálogo para la estrategia de los gobiernos en el cumplimiento del artículo 19 de la Convención de los derechos de las personas con discapacidad (Parker, 2011)

1. Comprometerse a la transformación del sistema desde los servicios institucionales a los servicios basados en la comunidad

2. Fomentar el reconocimiento explícito del derecho a la vida en comunidad para todos (el derecho de todas las personas con discapacidad a vivir en la comunidad, "con opciones iguales a los demás")

3. Desarrollar una estrategia nacional para la transformación del sistema de internamientos institucionales hacia los servicios y apoyos basados en la comunidad

4. Establecer mecanismos que permitan la participación de la sociedad civil, en particular, de las personas con discapacidad y sus familias

5. Desarrollar vínculos con expertos (internacionales y nacionales)

6. Revisión de la legislación, las políticas y las prácticas pertinentes para la aplicación del artículo 19

7. Revisión de los servicios existentes para las personas con discapacidad

8. Asegurar la transparencia y la rendición de cuentas en el uso de fondos públicos

9. Establecer mecanismos para la recopilación de datos

10. Establecer mecanismos para la revisión periódica del plan de acción y estrategia

Por otro lado, en un informe de Inclusion International (2012) se señala como problema para que las personas sean protagonistas en los procesos de toma de decisión sobre aspectos como su derecho a vivir en comunidad la "Falta de modelos e infraestructura para la toma de decisiones con apoyo" (p. 106), indicando como dirección futura la "Inversión en iniciativas piloto, legislación modelo y apoyos individualizados para elaborar buenas prácticas en la toma de decisiones con apoyo” (p. 106). También afirma:

Debemos aprender de nuestros errores, no repetirlos. La desinstitucionalización debe ser mucho más que el mero cierre de grandes instituciones o su reemplazo por otras más pequeñas; mucho más que la creación de redes de hogares grupales y, en definitiva, mucho más que la sustitución del aislamiento fuera de la comunidad por el aislamiento dentro de ella. La desinstitucionalización debe consistir en crear capacidad en la comunidad a fin de apoyar a las personas con discapacidad intelectual y sus familias para que vivan como ciudadanos plenos, en igualdad de condiciones con las demás personas, de una manera que refleje y respete la cultura y las tradiciones vigentes. El resultado debe ser lograr vidas comunes, pero valoradas (Inclusion International, 2012: 128).

También en el informe de Mansell y Beadle-Brown (2011) se evidencia que "El éxito de la atención comunitaria requiere prestar una especial atención a la forma en que se crean y gestionan los servicios, especialmente a la calidad del apoyo que presta el personal" (p. 143).

Y en el informe de la Dirección General de Empleo, Asuntos sociales e Igualdad (2011), de la Comisión Europea, se proponen entre otras medidas la de fomentar y facilitar el intercambio de modelos de buenas prácticas, contar con expertos que orienten el proceso y promover la formación de los profesionales de atención directa y de los gestores de los servicios existentes. 


\section{Por qué FEAPS habla de transformación}

FEAPS, movimiento asociativo español en favor de las personas con discapacidad intelectual o del desarrollo y de sus familias (www.feaps.org), lleva ya tiempo desarrollando un sistema de buenas prácticas para avanzar en el proceso de transformación cultural hacia el modelo de calidad de vida y de derechos (Tamarit, 2012) en coherencia con su misión. La misión del movimiento asociativo FEAPS es:

contribuir, desde su compromiso ético, con apoyos y oportunidades, a que cada persona con discapacidad intelectual o del desarrollo y su familia puedan desarrollar su proyecto de calidad de vida, así como a promover su inclusión como ciudadana de pleno derecho en una sociedad justa y solidaria (FEAPS, 2010: 1).

Esta misión orienta el 4. ${ }^{\circ}$ Plan Estratégico, actualmente en su último año, cuyo despliegue se realiza a través de proyectos estratégicos, y uno de ellos, el proyecto estratégico 4, es el denominado de Transformación hacia la calidad de vida, cuyo objetivo esencial es transformar la realidad de los centros y servicios para reorientarlos a la persona con discapacidad intelectual o del desarrollo, con el fin de contribuir a la realización de su proyecto de vida y al desarrollo de una sociedad más justa y solidaria. El sentido, por tanto, de este proyecto al que se refiere este artículo es orientar a las entidades, con sus servicios de apoyo, a una excelencia centrada en la persona y capaz de contribuir al desarrollo de una sociedad más justa y solidaria, promoviendo la implantación de calidad FEAPS, en línea con lo que se considera que debe ser la calidad en el sector social (Tamarit, 2010).

Este proyecto tiene dos grandes ramas: por un lado, la calidad FEAPS y su Sistema de Evaluación de la Calidad FEAPS (Tamarit, 2006; Grande, Hernández y Tamarit, 2015) y, por otro lado, el desarrollo de Servicios Centrados en la Persona. En estos momentos se está ultimando la edición de un documento explicativo y formativo sobre qué es para FEAPS un servicio centrado en la persona y un entorno Web que facilite la comprensión y el intercambio sobre esta conceptualización. A su vez, los 104 proyectos concretos de transformación que se están generando actualmente (Atención Temprana, Buena Vejez, Centros Ocupacionales, Educación...) entran en esta segunda rama del proyecto, que es el motivo del presente artículo.

Además, este proyecto tiene una base institucional y estratégica clara. En primer lugar, es un mandato de la Asamblea de FEAPS tras la aprobación del 4. ${ }^{\circ}$ Plan Estratégico que surgió del proceso de reflexión de todo el movimiento asociativo y que culminó en la ciudad de Toledo en 2010. En segundo lugar, en la denominada Declaración de Alcalá (FEAPS, 2013) se ratifica el compromiso con el avance de nuestros servicios y se nos insta a dar respuesta a nuevas necesidades y hacerlo de una manera más participativa e inclusiva. Y, por último, en tercer lugar, entre las líneas prioritarias asumidas por la Junta Directiva de FEAPS en junio de 2014 está la de la apuesta por los procesos de transformación de servicios centrados en las personas y su calidad de vida.

La idea de base es que en el conjunto del movimiento asociativo tenemos una red de centros y servicios que fueron creados en un momento en que el conocimiento que teníamos y la mirada que proyectábamos estaban centrados en la discapacidad, 
en el déficit. Y eso fue importante, pues anteriormente a ello prácticamente no existía nada. Pero siempre hemos estado implicados en mejorar lo que hacemos y eso nos ha permitido avanzar. Afortunadamente, y gracias también a los profesionales y responsables de la propia red de entidades, centros y servicios, estamos aprendiendo desde hace un tiempo a mirar y conocer de otra forma, a poner a la persona y a su familia en el centro de nuestras miradas y de nuestros esfuerzos en cada servicio de apoyo. Estos servicios, por tanto, fueron creados en el seno de una cultura del déficit y ahora se requiere su transformación hacia la cultura de la persona y su familia, de la ciudadanía, de la calidad de vida en contextos comunitarios inclusivos (Tamarit, 2014).

\section{La transformación: un proceso de innovación social en el marco de los derechos de las personas con discapacidad}

Este proceso de transformación no es exclusivo de nuestro entorno. En todo el mundo se está produciendo una transformación profunda en la visión de lo que la sociedad debe desarrollar en relación con las necesidades de las personas con discapacidad y del papel que a las propias personas con discapacidad les corresponde como voz colectiva para su empoderamiento y como voz singular para el desarrollo de su proyecto de vida personal, familiar y comunitario y del avance hacia su plena ciudadanía. Especialmente también la convención de los derechos de las personas con discapacidad (en España ver Boletín Oficial del Estado, 2008) ha venido a urgir este proceso de transformación, incluyendo la necesaria transformación del entramado legal, que obligadamente ha de acomodarse a los principios y derechos recogidos en dicha Convención.

La Convención, por ejemplo, dispone en su artículo 19 el derecho a vivir de forma independiente y a ser incluido en la comunidad. En coherencia con este artículo 19 se señala (Inclusion International, 2012) la necesidad de comenzar procesos de desinstitucionalización y avanzar hacia servicios incluidos en la comunidad, entendiendo la inclusión en la comunidad más allá del tipo de servicio, como un contexto que promueve la participación, la contribución, la inclusión, ofrece apoyos y relaciones significativas y permite la elección y la toma de decisiones. Así, Inclusion International (2012) plantea la necesidad de desarrollar lo que denomina desinstitucionalización de segundo orden, es decir, la transformación de los servicios comunitarios actuales, creados en gran parte por movimientos de familias, tales como FEAPS. Como se indica en ese informe:

El desafío de los líderes de las organizaciones de familias, incluidos los autogestores, es ayudar a transformar los servicios y apoyos de bajo valor, segregados y aislantes en apoyos y servicios de alto valor que ayuden a cumplir las promesas de la Convención de los Derechos de las Personas con Discapacidad y, en especial, de su artículo 19. Se corren riesgos al permanecer en lo que conocemos bien y reproducir modelos de servicios del pasado. Si las organizaciones de familias no dan el ejemplo, otros proveedores de servicios pueden tomar la iniciativa, debilitando así las voces de las familias. Si las organizaciones de familias no toman la iniciativa de asegurar que las personas con discapacidad tengan una vida de inclusión plena, es muy posible que pierdan parte de su autoridad moral y la capacidad de influir en los cambios de otros proveedores de servicios 
y de los gobiernos. Las personas con discapacidad intelectual y sus familias no pueden permitirse que eso suceda. Las organizaciones de familias deben generar la visión y la pasión por la transformación, tanto para las propias organizaciones de familias como para los proveedores de servicios y los gobiernos. Se trata de algo demasiado importante como para dejarlo librado al azar (Inclusion International, 2012: 122).

El artículo 12 de la Convención, sobre el derecho a decidir, exige también la transformación de los apoyos y la inversión en organizaciones que puedan ayudar a desarrollar y fortalecer la inclusión en la comunidad. Inclusion International (2014) hace, entre otras, las siguientes recomendaciones: "Reconvertir las opciones de vivienda, empleo y otros servicios y apoyos segregados que reúnen a personas con discapacidad intelectual en apoyos inclusivos e individualizados mediante la planificación centrada en la persona” (p. 121). Dentro de propuestas para una agenda común para lograr la efectividad del derecho a decidir se ofrece, entre otras muchas, la siguiente idea para los gobiernos: "Favorecer la reconversión de servicios y apoyos segregados en apoyos inclusivos e individualizados” (p. 124).

España, tras ratificar la Convención, está concernida en cuanto que los Estados parte que la ratifican:

reconocen el derecho en igualdad de condiciones de todas las personas con discapacidad a vivir en la comunidad, con opciones iguales a las de las demás, y adoptarán medidas efectivas y pertinentes para facilitar el pleno goce de este derecho por las personas con discapacidad y su plena inclusión y participación en la comunidad (Boletín Oficial del Estado, 2008: 20653).

Por otro lado, las políticas europeas, dentro del marco de la estrategia europea 2020, promueven la innovación como un factor crítico para un desarrollo sostenible y consideran, dentro del plan de unión por la innovación, que esta iniciativa conducirá a una serie de avances que, entre otros impactos, mejorarán la calidad de vida y que se dará "poder a los ciudadanos gracias a la innovación social” (Comisión Europea, 2013: 12), lo que son dos ámbitos esenciales de los modelos que guían el proyecto de transformación en FEAPS.

También en el sector público se considera que se ha de dar un giro para además de alimentar y promover la innovación en los demás sectores, incluido el sector social, incorporar la cultura de la innovación dentro de los propios procesos y servicios públicos. Así, se indica lo siguiente:

La innovación en el sector público (...) se puede definir como el proceso de generación de nuevas ideas y ponerlas en práctica para crear valor para la sociedad, lo que abarca los procesos nuevos o mejorados (enfoque interno) y los servicios (enfoque externo). Toma una variedad de formas, que van desde la compra inteligente, movilizando nuevas formas de financiación de la innovación, la creación de plataformas digitales y de servicios centrados en los ciudadanos, así como la conducción de una nueva cultura emprendedora entre los gestores públicos (Bason, 2013: 5) (el resaltado es nuestro).

Hay además una alta vinculación entre el desarrollo de procesos de innovación social y el avance en el cumplimiento de los objetivos temáticos de la estrategia europea 
2020, especialmente con el objetivo temático 9 referido a promover la inclusión social y luchar contra la pobreza y cualquier forma de discriminación.

A su vez, la Estrategia Española sobre Discapacidad 2012-2020 (Ministerio de Sanidad, Política Social e Igualdad, 2011) es un marco político y de acción que contempla, entre otros, los principios inspiradores de no discriminación, igualdad de oportunidades, vida independiente, accesibilidad universal y participación, la mayor parte de los cuales coinciden como objetivos principales de la estrategia. Además contempla como medida estratégica "Promover el refuerzo de la $\mathrm{I}+\mathrm{D}+\mathrm{i}$ de discapacidad en Estrategia Española de Innovación mediante programas específicos” (Ministerio de Sanidad, Política Social e Igualdad, 2011: 30).

\section{La experiencia de FEAPS en la transformación de los servicios}

Desde 2010 en FEAPS estamos trabajando, como compromiso estratégico, en la conceptualización y las características que debe tener un Servicio Centrado en la Persona (González, Ortega, Martínez y Tamarit, 2014) y estamos desarrollando experiencias concretas de transformación. También se han desarrollado acciones de difusión y conocimiento del proyecto; se puede ver una de estas acciones en video en la Web (AGORA, 2014, octubre 14). En esta jornada se explicó el proyecto, debatieron gerentes de servicios sobre su visión y su práctica en procesos de transformación y también se presentaron experiencias concretas de transformación.

\section{Qué es un servicio centrado en la persona}

La Figura 2 muestra las tres características que consideramos en FEAPS irrenunciables en un Servicio Centrado en la Persona y que se detallan a continuación:

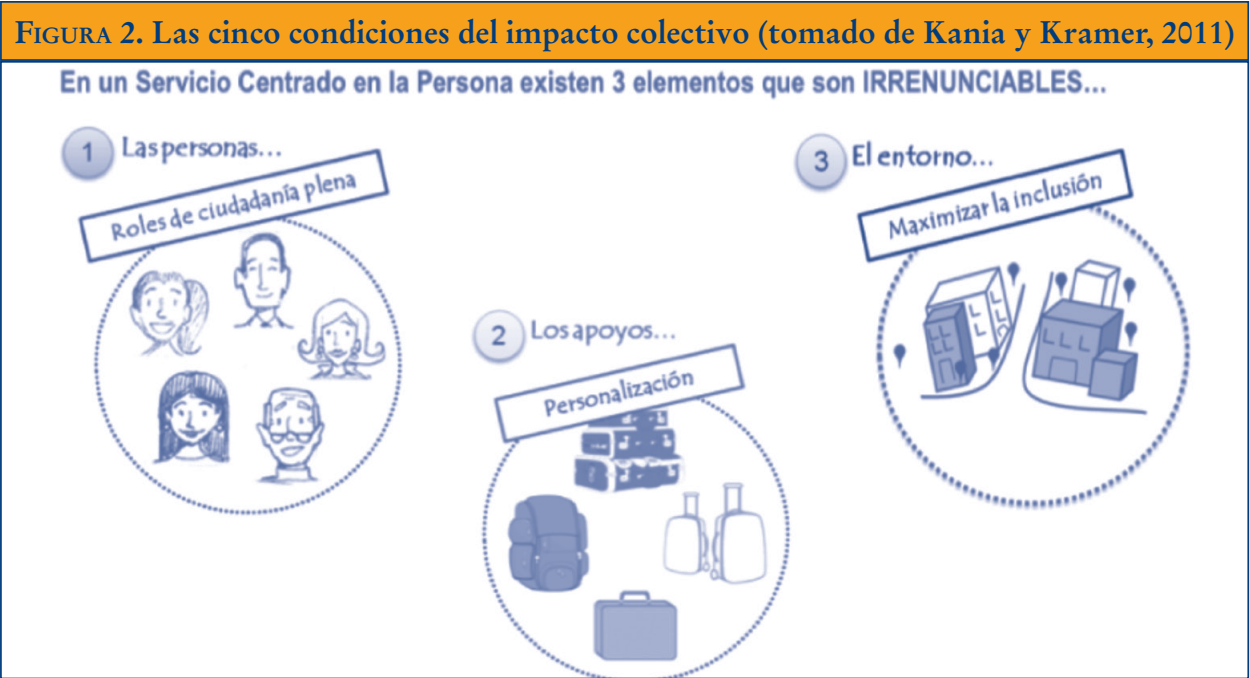

(C) Ediciones Universidad de Salamanca

Siglo Cero, vol. 46 (3), n. ${ }^{\circ} 255,2015$, julio-septiembre, pp. 47-71 
El ejercicio de roles de ciudadanía plena en todas personas. Con un liderazgo de las propias personas con discapacidad intelectual, que deben tener poder para el desarrollo de su proyecto de vida, para contribuir como ciudadanos a una sociedad mejor y para la reivindicación y el ejercicio, como colectivo, de sus derechos y de su ciudadanía. No se trata únicamente de diseñar acciones para que la persona logre sus metas personales, sino generar un cambio de rol en las personas con discapacidad, como receptoras de apoyos a ciudadanos de pleno derecho, en el que lideran su propio proyecto de vida, se responsabilizan de generar cambios en su entorno cercano como colectivo y entienden la necesidad de contribuir a una sociedad diferente. El Servicio Centrado en la Persona debe contemplar a la persona desde esta triple perspectiva prestando apoyos y oportunidades para favorecer un empoderamiento total en los tres ámbitos.

A su vez las familias deben ser cómplices en la transformación, alineando su proyecto de vida familiar con el proyecto de la persona con discapacidad del desarrollo, con lo que el Servicio Centrado en la Persona solo puede ser así si también se entiende como Servicio Centrado en la Familia (quizá deberíamos hablar de Servicio Centrado en la Persona, en general, entendiendo por Persona a cualquiera que sea la receptora de los servicios de apoyo que se ofrecen, tanto si es una persona con discapacidad intelectual o del desarrollo, un familiar, una familia o alguna otra persona de la red natural o comunitaria de apoyos). En relación a la familia, por tanto, se debe trabajar desde las fortalezas y características particulares de cada familia como sistema en un planteamiento centrado en la mejora de su calidad de vida familiar. Así mismo se entiende a los familiares como generadores de oportunidades en su entorno comunitario y proporcionando oportunidades de participación dentro de la propia familia y en el entorno.

El rol de los profesionales es un rol comprometido. Pasa de mero prestador de apoyos en entornos segregados, a identificador activo de oportunidades de participación en contextos inclusivos y a prestador de apoyos en entornos comunitarios. Los profesionales, como profesionales de la personalización en el entorno comunitario, deben estar comprometidos con la persona y con la familia, generando pensamiento, conocimiento y apoyos centrados en la persona y en su familia, e identificando y promoviendo oportunidades y apoyos en el entorno de cada persona. Los profesionales de un Servicio Centrado en la Persona se sienten vinculados a las personas y son agentes de transformación de los barrios donde estas viven, ya no solo se vinculan a los centros específicos y a sus programas.

La personalización en los sistemas de apoyo. Cada persona ha de tener un sistema integral y flexible de apoyos en base a los resultados personales que persiga. Un eficaz sistema de apoyos personalizados ayuda a la persona a controlar lo que pasa en su vida, genera procesos de escucha e indagación constantes, alienta el pensamiento de la persona y de su red de apoyos, apoya el desarrollo de competencias de cada persona a través de prácticas basadas en la evidencia científica y en la ética, mantiene a la persona con sus apoyos naturales y los dinamiza, se produce a través de presupuestos personalizados (no sólo en costes/plaza) (Morgan, 2010) y cuenta con un sistema de planificación, ejecución y evaluación en relación con los resultados personales que se 
persiguen. Por lo tanto las organizaciones podríamos decir que han de pasar a ser organizaciones líquidas, dejar de ser sólidas, en el sentido metafórico de dejar de centrar las miradas en los 'ladrillos', en las estructuras y pasar a centrar la mirada en algo menos sólido, más líquido, más escurridizo entre las manos organizacionales, como son las vidas de las personas. Las organizaciones han de ser, ahora, más bandas de jazz que orquestas sinfónicas. Esto implica un modelo de relación entre los profesionales y sus organizaciones, un modelo centrado en la profesionalidad, en el sentido que se señala en la conceptualización de la discapacidad intelectual propuesta en la 11. a edición de la AAIDD (Schalock et al., 2010).

Mantiene a la persona conectada con su entorno y lo transforma, maximiza la inclusión y las oportunidades de participación y desarrollo comunitario. Trabaja en el entorno $y$ desde el entorno. Colabora en la transformación hacia una comunidad inclusiva y empoderada. Dispone de estrategias para cultivar las redes de apoyo natural de cada persona. Identifica y promueve el valor con el que cada persona puede contribuir a la comunidad y la apoya en el ejercicio de un rol social valorado. Lidera y apoya la transformación hacia un entorno inclusivo con oportunidades para todas las personas. Entiende a la persona desde su contexto natural y apoya una cultura que fomenta la ciudadanía en todas las personas.

En definitiva, es un servicio con una postura claramente transformadora de su entorno, que alienta una sociedad civil comprometida, que trata de aportar y desarrollar acciones para lograr un entorno de oportunidades para todos, trabajando por y para la comunidad y no solo reivindicando para el colectivo mejores prestaciones o ayudas económicas. Este es un servicio que observa dificultades y aporta soluciones, que reivindica el cumplimiento de derechos y que abre sus puertas a la colaboración de personas que compartan su misión y objetivos poniendo su aportación en valor.

Existen además, en este modelo de FEAPS, 5 claves que ayudan a los procesos de transformación hacia Servicios Centrados en las Personas, estas son:

1. Promover un liderazgo visionario y diverso.

2. Compartir valores y creencias en el día a día.

3. Contar con un diseño y estructura organizacional flexible y fluida.

4. Practicar la capacidad de aprendizaje individual y organizacional.

5. Tejer alianzas con otros.

Veamos a continuación algunos datos sobre los proyectos de transformación que FEAPS está ya desarrollando en los ámbitos de la atención temprana, la educación, los centros ocupacionales y los servicios para personas mayores con discapacidad intelectual o del desarrollo (en total están implicados actualmente 104 servicios de 13 comunidades autónomas diferentes: Galicia, País Vasco, Cataluña, Castilla y León, La Rioja, Madrid, Comunidad Valenciana, Islas Baleares, Extremadura, Castilla la Mancha, Andalucía, Región de Murcia, Islas Canarias). 


\section{Proyecto de Transformación 'La Atención Temprana que Queremos'}

Actualmente, hay 38 servicios de atención temprana de 10 comunidades diferentes que están trabajando en proyectos de transformación para orientar la atención temprana a los modelos que FEAPS propone de servicios centrados en la persona. En el total de esos 38 servicios hay 376 profesionales que atienden a 4596 familias.

En atención temprana la propuesta de servicio coherente con lo que FEAPS propugna es una propuesta comprensiva integradora de atención y apoyos basados y centrados en:

- el conocimiento sobre el desarrollo humano y sobre sus alteraciones

- el uso de prácticas basadas en la evidencia y en la ética

- enfoques centrados en la familia (incluido el niño)

- el logro de mayor bienestar familiar

- el logro de mayor bienestar y desarrollo personal

- la intervención en el contexto natural

- acciones para contribuir a la generación de contextos protectores e inclusivos.

Tras una primera fase de este proyecto, 22 de los 38 servicios de atención temprana expusieron sus avances en sus proyectos de transformación en una jornada celebrada en Madrid. Las ponencias están disponibles en video en la Web (FEAPS CONFEDERACION, 2014, diciembre 15 y 16).

Los servicios que presentaron avances en sus proyectos de transformación hacia 'La atención Temprana que queremos' se pueden ver en la Tabla 4.

\begin{tabular}{|l|l|l|}
\hline \multicolumn{3}{|c|}{$\begin{array}{c}\text { TABLA 4. Servicios de Atención Temprana que han evidenciado sus avances } \\
\text { en proyectos de transformación }\end{array}$} \\
\hline Centros de Atención Temprana & \multicolumn{1}{|c|}{ Entidad } & Comunidad Autónoma \\
\hline CDIAT Adislan & ADISLAN & Islas Canarias \\
\hline CDIAT AFAS Alcázar de San Juan & AFAS & Castilla la Mancha \\
\hline CDIAT Bolaños & AFAS & Castilla la Mancha \\
\hline CDIAT Socuéllamos & AFAS & Castilla la Mancha \\
\hline CDIAT Tomelloso & AFAS & Castilla la Mancha \\
\hline Saicon Aleph-Tea & Aleph-Tea & Madrid \\
\hline Centro San Leandro & ASIDO Cartagena & Murcia \\
\hline CDIAT ASODEMA & ASODEMA & Castilla la Mancha \\
\hline ASPACE Gipuzkoa & ASPACE & País Vasco \\
\hline CDIAT Campo Criptana & ASPANA & Castilla la Mancha \\
\hline SEDIAP Aproscom Fundació & Asprocom Fundació & Islas Baleares \\
\hline CDIAT Asprona Adapei & ASPRONA Albacete & Castilla la Mancha \\
\hline CDIAT Asprona Almansa & ASPRONA Albacete & Castilla la Mancha \\
\hline
\end{tabular}




\begin{tabular}{|l|l|l|}
\hline \multicolumn{3}{|c|}{ TABLA 4. Servicios de Atención Temprana que han evidenciado sus avances } \\
en proyectos de transformación
\end{tabular}

En la Tabla 5 se muestran los 38 servicios incluidos en el proyecto de transformación.

\begin{tabular}{|c|c|c|}
\hline Centros de Atención Temprana & Entidad & Federación \\
\hline $\begin{array}{l}\text { CDIAT Adislan. Arrecife, Lanzarote, } \\
\text { Las Palmas }\end{array}$ & ADISLAN & FEAPS CANARIAS \\
\hline CDIAT Afad. Valdepeñas, Ciudad Real & AFAD & $\begin{array}{l}\text { FEAPS CASTILLA LA } \\
\text { MANCHA }\end{array}$ \\
\hline $\begin{array}{l}\text { CDIAT AFAS Alcázar de San Juan, } \\
\text { Ciudad Real }\end{array}$ & AFAS & $\begin{array}{l}\text { FEAPS CASTILLA LA } \\
\text { MANCHA }\end{array}$ \\
\hline CDIAT AFAS Bolaños, Ciudad Real & AFAS & $\begin{array}{l}\text { FEAPS CASTILLA LA } \\
\text { MANCHA }\end{array}$ \\
\hline \begin{tabular}{|l} 
CDIAT AFAS Socuéllamos, Ciudad \\
Real \\
\end{tabular} & AFAS & $\begin{array}{l}\text { FEAPS CASTILLA LA } \\
\text { MANCHA } \\
\end{array}$ \\
\hline CDIAT AFAS Tomelloso, Ciudad Real & AFAS & $\begin{array}{l}\text { FEAPS CASTILLA LA } \\
\text { MANCHA }\end{array}$ \\
\hline Saicón ALEPH TEA, Madrid & ALEPH-TEA & FEAPS MADRID \\
\hline CAT Apansa. Alcorcón, Madrid & APANSA & FEAPS MADRID \\
\hline $\begin{array}{l}\text { Servicio Especializado de Atención } \\
\text { Temprana. Alcalá de Henares, Madrid }\end{array}$ & APHISA & FEAPS MADRID \\
\hline $\begin{array}{l}\text { Servei de desenvolupament infantil i } \\
\text { atenció primaria. Manacor, Palma de } \\
\text { Mallorca }\end{array}$ & APROSCOM & $\begin{array}{l}\text { FEAPS ISLAS } \\
\text { BALEARES }\end{array}$ \\
\hline $\begin{array}{l}\text { CAT Aprosuba 9. Villanueva de la } \\
\text { Serena, Badajoz }\end{array}$ & APROSUBA 9 & $\begin{array}{l}\text { FEAPS } \\
\text { EXTREMADURA }\end{array}$ \\
\hline $\begin{array}{l}\text { CAT José Espinosa Bote. Villafranca de } \\
\text { los Barros, Badajoz }\end{array}$ & APROSUBA 13 & $\begin{array}{l}\text { FEAPS } \\
\text { EXTREMADURA }\end{array}$ \\
\hline $\begin{array}{l}\text { Centro San Leandro. Cartagena, } \\
\text { Murcia } \\
\end{array}$ & ASIDO CARTAGENA & $\begin{array}{l}\text { FEAPS REGIÓN DE } \\
\text { MURCIA }\end{array}$ \\
\hline
\end{tabular}




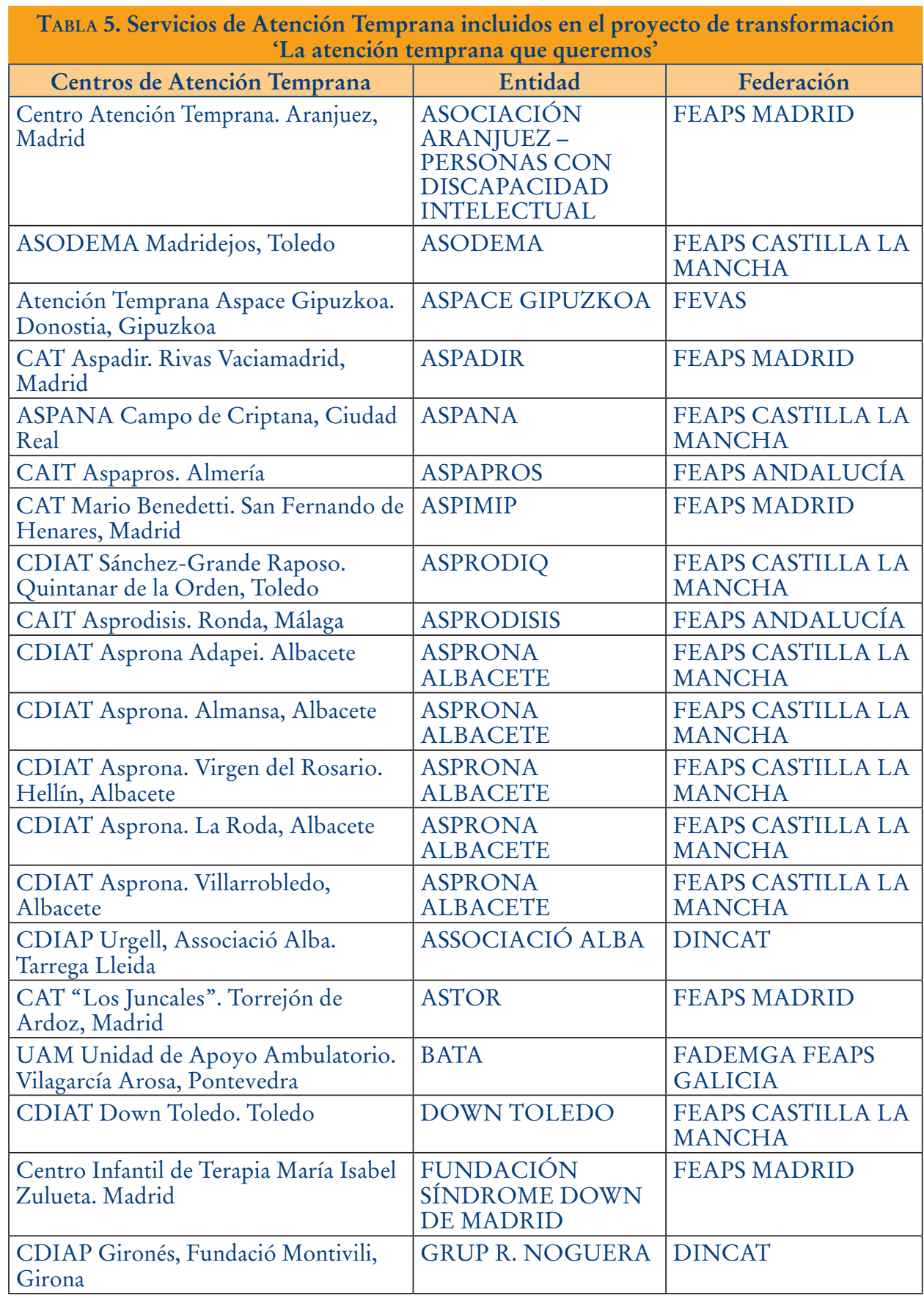




TABLA 5. Servicios de Atención Temprana incluidos en el proyecto de transformación
'La atención temprana que queremos'
\begin{tabular}{|l|l|l|}
\hline \multicolumn{1}{|c|}{ Centros de Atención Temprana } & \multicolumn{1}{|c|}{ Entidad } & \multicolumn{1}{c|}{ Federación } \\
\hline GRUPO AMÁS. Móstoles, Madrid & GRUPO AMÁS & FEAPS MADRID \\
\hline $\begin{array}{l}\text { CDIAP Baix Penedés, Associació } \\
\text { L'Espiga Penedés, El Vendrell, Tarragona }\end{array}$ & $\begin{array}{l}\text { L'ESPIGA CENTRO } \\
\text { DE VENDRELL }\end{array}$ & DINCAT \\
\hline $\begin{array}{l}\text { CDIAP Vilafranca del Penedés. } \\
\text { Associaciò L'Espiga Penedés, } \\
\text { Vilafranca, Barcelona }\end{array}$ & $\begin{array}{l}\text { L'ESPIGA CENTRO } \\
\text { DE VILAFRANCA }\end{array}$ & DINCAT \\
\hline CAIT Prode. Pozoblanco, Córdoba & PRODE & FEAPS ANDALUCÍA \\
\hline $\begin{array}{l}\text { CDIAP Tris Tras Associació Sant } \\
\text { Tomas, Vic }\end{array}$ & SANT TOMÁS & DINCAT \\
\hline
\end{tabular}

'La educación que queremos'. Proyecto de Transformación de Centros especificos de educación especial

FEAPS ha establecido en su propuesta 'La educación que queremos' (para todo el alumnado, con o sin necesidades educativas especiales) la siguiente misión de la educación: "conseguir que las personas tengan poder para, con los apoyos necesarios, construir, desplegar y defender su proyecto vital en contextos de convivencia justos, contribuyendo a la riqueza humana y a la transformación hacia una sociedad de excelencia moral" (FEAPS, 2009: 38).

El proyecto de transformación 'La educación que queremos' tiene como objetivo avanzar hacia esa misión también en los centros de educación especial. En la Tabla 6 se muestran los centros que se han comprometido voluntariamente con este proyecto de transformación y que ya están trabajando en el mismo (la primera fase de este proyecto finaliza en el último trimestre de 2016).

TABLA 6. Centros de educación especial que se han comprometido con el proyecto de transformación ' $\mathrm{La}$ educación que queremos'

\begin{tabular}{|l|l|l|}
\hline \multicolumn{1}{|c|}{ Entidad } & \multicolumn{1}{|c|}{ Federación } & \multicolumn{1}{c|}{ Centro Educación Especial } \\
\hline A.R.P.S. & FEAPS LA RIOJA & CPCEE “Los Ángeles” \\
\hline ALEPH-TEA & FEAPS MADRID & $\begin{array}{l}\text { Colegio de Educación Especial } \\
\text { ALEPH }\end{array}$ \\
\hline APACE & $\begin{array}{l}\text { FEAPS CASTILLA LA } \\
\text { MANCHA }\end{array}$ & Nuestra Señora del Prado \\
\hline APROSUB & FEAPS ANDALUCÍA & CEE María Montessori de Córdoba \\
\hline APROSUB & FEAPS ANDALUCÍA & $\begin{array}{l}\text { CEE “EL MOLINILLO } \\
\text { APROSUB-MONTILLA" }\end{array}$ \\
\hline APROSUB & FEAPS ANDALUCÍA & $\begin{array}{l}\text { CDPEE Nuestra Señora del } \\
\text { Rosario }\end{array}$ \\
\hline APROSUB & FEAPS ANDALUCÍA & CEE Manuel Benítez \\
\hline
\end{tabular}




\begin{tabular}{|c|c|c|}
\hline \multicolumn{3}{|c|}{$\begin{array}{c}\text { TABLA 6. Centros de educación especial que se han comprometido con el proyecto } \\
\text { de transformación 'La educación que queremos' }\end{array}$} \\
\hline Entidad & Federación & Centro Educación Especial \\
\hline APROSUB & FEAPS ANDALUCÍA & $\begin{array}{l}\text { CEE María Montessori-Aprosub } \\
\text { Castro del Río }\end{array}$ \\
\hline APROSUBA 4 & $\begin{array}{l}\text { FEAPS } \\
\text { EXTREMADURA }\end{array}$ & CEE "Ntra. Señora de las Cruces" \\
\hline $\begin{array}{l}\text { Asociación "Virgen del } \\
\text { Valle" APACE }\end{array}$ & $\begin{array}{l}\text { FEAPS CASTILLA LA } \\
\text { MANCHA }\end{array}$ & CEE “San Juan de Dios” \\
\hline $\begin{array}{l}\text { Asociación ASPADES - La } \\
\text { Laguna }\end{array}$ & $\begin{array}{l}\text { FEAPS CASTILLA LA } \\
\text { MANCHA }\end{array}$ & CCEE ASPADES - La Laguna \\
\hline Asociación BATA & $\begin{array}{l}\text { FADEMGA FEAPS } \\
\text { GALICIA }\end{array}$ & CEEPR BATA OS MECO \\
\hline Asociación Juan XXIII & $\begin{array}{l}\text { FADEMGA FEAPS } \\
\text { GALICIA }\end{array}$ & Colegio Juan XXIII \\
\hline $\begin{array}{l}\text { Asociación Pro Deficientes } \\
\text { Psíquicos Jerome Lejeune }\end{array}$ & $\begin{array}{l}\text { FEAPS CASTILLA LA } \\
\text { MANCHA }\end{array}$ & CCEE Jerome Lejeune \\
\hline ASPANA & $\begin{array}{l}\text { FEAPS CASTILLA LA } \\
\text { MANCHA }\end{array}$ & $\begin{array}{l}\text { Centro Comarcal de Educación } \\
\text { Especial "María Auxiliadora" }\end{array}$ \\
\hline $\begin{array}{l}\text { ASPRODES FEAPS } \\
\text { Salamanca }\end{array}$ & $\begin{array}{l}\text { FEAPS CASTILLA Y } \\
\text { LEÓN }\end{array}$ & $\begin{array}{l}\text { Centro Concertado de Educación } \\
\text { Especial "El Arca" }\end{array}$ \\
\hline ASPRODIQ & $\begin{array}{l}\text { FEAPS CASTILLA LA } \\
\text { MANCHA }\end{array}$ & CCEE Stmo. Cristo de la Salud \\
\hline ASPRODIS & $\begin{array}{l}\text { FEAPS } \\
\text { EXTREMADURA }\end{array}$ & CEE Nuestra Señora de la Luz \\
\hline ASPRONA ALBACETE & $\begin{array}{l}\text { FEAPS CASTILLA LA } \\
\text { MANCHA }\end{array}$ & $\begin{array}{l}\text { CCEE “ASPRONA” } \\
\text { (ALMANSA) }\end{array}$ \\
\hline ASPRONA ALBACETE & $\begin{array}{l}\text { FEAPS CASTILLA LA } \\
\text { MANCHA }\end{array}$ & $\begin{array}{l}\text { CCEE Infanta Elena } \\
\text { (VILLAROBLEDO) }\end{array}$ \\
\hline ASPRONA ALBACETE & $\begin{array}{l}\text { FEAPS CASTILLA LA } \\
\text { MANCHA }\end{array}$ & $\begin{array}{l}\text { CEE “Virgen de los Remedios" } \\
\text { (LA RODA) }\end{array}$ \\
\hline \begin{tabular}{|l|} 
ASPRONA \\
VALLADOLID \\
\end{tabular} & $\begin{array}{l}\text { FEAPS CASTILLA Y } \\
\text { LEÓN } \\
\end{array}$ & El Pino de Obregón \\
\hline ASPROSUBA 8 & $\begin{array}{l}\text { FEAPS } \\
\text { EXTREMADURA }\end{array}$ & CEE Ponce de León \\
\hline ASTOR & FEAPS MADRID & VIRGEN DE LORETO-REHTO \\
\hline $\begin{array}{l}\text { CENTRO SAN JUAN DE } \\
\text { DIOS }\end{array}$ & $\begin{array}{l}\text { FEAPS CASTILLA Y } \\
\text { LEÓN }\end{array}$ & $\begin{array}{l}\text { CEE San Juan de Dios de } \\
\text { Valladolid }\end{array}$ \\
\hline Fundación ADEMO & FEAPS MADRID & CEE ADEMO \\
\hline Fundación AMPANS & DINCAT & $\begin{array}{l}\text { Escola d'Educació Especial Jeroni } \\
\text { de Moragas }\end{array}$ \\
\hline Fundación Aspanias Burgos & $\begin{array}{l}\text { FEAPS CASTILLA Y } \\
\text { LEÓN }\end{array}$ & $\begin{array}{l}\text { Centro Concertado de Educación } \\
\text { Especial Puentesauco }\end{array}$ \\
\hline
\end{tabular}


TABLA 6. Centros de educación especial que se han comprometido con el proyecto de transformación 'La educación que queremos'

\begin{tabular}{|l|l|l|}
\hline \multicolumn{1}{|c|}{ Entidad } & \multicolumn{1}{|c|}{ Federación } & \multicolumn{1}{c|}{ Centro Educación Especial } \\
\hline Fundación Gil Gayarre & FEAPS MADRID & CCEE Fundación Gil Gayarre \\
\hline $\begin{array}{l}\text { Fundación Madre de la } \\
\text { Esperanza de Talavera de la } \\
\text { Reina }\end{array}$ & $\begin{array}{l}\text { FEAPS CASTILLA LA } \\
\text { MANCHA }\end{array}$ & $\begin{array}{l}\text { CCEE Madre de la Esperanza de } \\
\text { Talavera de la Reina }\end{array}$ \\
\hline Fundación San Cebrián & $\begin{array}{l}\text { FEAPS CASTILLA Y } \\
\text { LEÓN }\end{array}$ & $\begin{array}{l}\text { Centro Educación Especial Canal } \\
\text { de Castilla }\end{array}$ \\
\hline $\begin{array}{l}\text { Fundación Síndrome de } \\
\text { Down Madrid }\end{array}$ & FEAPS MADRID & CEE Carmen Fernández-Miranda \\
\hline $\begin{array}{l}\text { Fundación Síndrome de } \\
\text { Down Madrid }\end{array}$ & FEAPS MADRID & CEE María Isabel Zulueta \\
\hline $\begin{array}{l}\text { Koynos Enseñanza CEE } \\
\text { Coop. Val. }\end{array}$ & $\begin{array}{l}\text { FEAPS C. } \\
\text { VALENCIANA }\end{array}$ & CEE KOYNOS \\
\hline $\begin{array}{l}\text { MATER } \\
\text { MISERICORDIAE }\end{array}$ & $\begin{array}{l}\text { FEAPS ISLAS } \\
\text { BALEARES }\end{array}$ & CEE Mater Misericordiae \\
\hline $\begin{array}{l}\text { Patronato Intermunicipal } \\
\text { Francisco Esteve }\end{array}$ & $\begin{array}{l}\text { FEAPS C. } \\
\text { VALENCIANA }\end{array}$ & CEE Francisco Esteve \\
\hline PAUTA & FEAPS MADRID & PAUTA \\
\hline $\begin{array}{l}\text { SAN XEROME } \\
\text { EMILIANI }\end{array}$ & $\begin{array}{l}\text { FADEMGA FEAPS } \\
\text { GALICIA }\end{array}$ & CEEPR San Xerome Emiliani \\
\hline
\end{tabular}

\section{Avanzando en la Transformación de los Centros Ocupacionales}

Este proyecto piloto de transformación se está desarrollando en FEAPS Madrid con la colaboración de la Confederación FEAPS. En cada centro se ha creado un equipo promotor del proyecto formado por personas con discapacidad intelectual o del desarrollo, familiares, profesionales de atención directa y responsables del servicio. En el conjunto de los centros se ofrece apoyo a 1.053 personas con discapacidad intelectual o del desarrollo.

En la Tabla 7 se muestran las entidades con centros ocupacionales en este proyecto.

\begin{tabular}{|l|l|}
\hline \multicolumn{1}{|c|}{ TABLA 7. Entidades con centros ocupacionales en el proyecto piloto de transformación } \\
\hline \multicolumn{1}{|c|}{ Entidad } & \multicolumn{1}{c|}{ Federación } \\
\hline FUNDACIÓN GIL GAYARRE & FEAPS MADRID \\
\hline AFANIAS & FEAPS MADRID \\
\hline CIRVITE & FEAPS MADRID \\
\hline FUNDACIÓN APROCOR & FEAPS MADRID \\
\hline GRUPO AMÁS (cuatro centros ocupacionales) & FEAPS MADRID \\
\hline ASTOR & FEAPS MADRID \\
\hline
\end{tabular}

(C) Ediciones Universidad de Salamanca

Siglo Cero, vol. 46 (3), n. ${ }^{\circ} 255,2015$, julio-septiembre, pp. 47-71 


\section{Proyecto Buena Vejez}

Tras una primera experiencia piloto con 10 personas mayores, hoy 116 personas con discapacidad intelectual o del desarrollo, mayores de 55 años, están implicadas en este proyecto. Se trata de orientar los programas y planes personales desde la biografía positiva de la persona y atendiendo a sus deseos, intereses y anhelos.

En la Fundación Gil Gayarre, pionera de este proyecto junto con FEAPS Madrid, se ha desarrollado este año una adaptación piloto para familiares y personas con necesidades complejas. Este programa cuenta con la colaboración del Ministerio de Sanidad, Servicios Sociales e Igualdad y la Universidad Complutense de Madrid. En la Tabla 8 se muestran las entidades participantes en este proyecto.

\begin{tabular}{|l|l|}
\hline \multicolumn{2}{|c|}{ TABLA 8. Entidades participantes en el proyecto de transformación 'Buena Vejez' } \\
\hline \multicolumn{2}{|c|}{ Entidad } \\
\hline AFANAS JEREZ (JEREZ DE LA FRONTERA - CÁDIZ) & FEAPS ANDALUCÍA \\
\hline APROSUB (CÓRDOBA) & FEAPS ANDALUCÍA \\
\hline ASOCIACIÓN PAZ Y BIEN & FEAPS ANDALUCÍA \\
\hline ASOCIACIÓN SAN JOSÉ (GUADIX - GRANADA) & FEAPS ANDALUCÍA \\
\hline ASPROGRADES (GRANADA) & FEAPS ANDALUCÍA \\
\hline ATUREM (SEVILLA) & FEAPS ANDALUCÍA \\
\hline FEAPS ANDALUCÍA & FEAPS ANDALUCÍA \\
\hline ASPRODES & FEAPS CASTILLA Y LEÓN \\
\hline FEAPS CASTILLA Y LEÓN & FEAPS CASTILLA Y LEÓN \\
\hline FUNDACIÓN ASPANIAS (BURGOS) & FEAPS CASTILLA Y LEÓN \\
\hline FUNDACIÓN PERSONAS (ZAMORA) & FEAPS CASTILLA Y LEÓN \\
\hline FUNDACIÓN SAN CEBRIÁN (PALENCIA) & FEAPS CASTILLA Y LEÓN \\
\hline APASA & DINCAT \\
\hline ASOCIACIÓN ALBA & DINCAT \\
\hline FUNDACIÓN FINESTRELLES (BARCELONA) & DINCAT \\
\hline AFANIAS TORRELAGUNA & FEAPS MADRID \\
\hline ASTOR & FEAPS MADRID \\
\hline FEAPS MADRID & FEAPS MADRID \\
\hline FUNDACIÓN ALAS (MORATALAZ) & FEAPS MADRID \\
\hline FUNDACIÓN GIL GAYARRE & FEAPS MADRID \\
\hline FUNDACIÓN MAGDALENA & FEAPS MADRID \\
\hline GRANJA SAN JOSÉ (FGG) & FEAPS MADRID \\
\hline GRUPO AMÁS & FEAPS MADRID \\
\hline
\end{tabular}


Claves que estamos aprendiendo para la transformación de los servicios hacia la calidad de vida

Emprender un viaje de transformación desde los servicios que tenemos hacia los servicios que se necesitan y que, éticamente, estamos comprometidos a ofrecer requiere de reflexión, conocimiento, compromiso y acción. Porque la transformación de nuestros servicios se asienta en una base de conocimiento científico pero también en una base de derechos de las personas y de sus familias. Y para ese viaje no partimos de cero, existen en los servicios muchas buenas prácticas y buen conocimiento experto acumulado a lo largo de más de 50 años de historia (Lacasta, 2015).

Por tanto, el proyecto de transformación de FEAPS consiste en alimentar y promover la voluntad y la acción de servicios de FEAPS que voluntariamente quieran iniciar un proceso de transformación y ofrecerles un entorno de red y de colaboración mutua, con apoyo y acompañamiento. Pero además hay una clave clara del proyecto: no nos da igual hacia dónde dirigirnos con este 'viaje', tenemos muy claro el modelo que orienta nuestra voluntad y acción transformadoras (modelo que ya se ha detallado en un apartado anterior, como Servicio Centrado en la Persona), un modelo centrado en la persona y en la familia y en el contexto natural (hogar, escuela, trabajo, servicios, comunidad), con la colaboración estrecha y en igualdad entre personas, familias y profesionales, con una base sólida en el uso de prácticas que cuenten con conocimiento y evidencia científicos sobre el funcionamiento humano y sobre las claves para su bienestar, tanto individual como familiar, y con un compromiso irrenunciable con los derechos de todas las personas.

La manera en que estamos desarrollando este proyecto en FEAPS se asienta en el conocimiento científico sobre el funcionamiento de las organizaciones que se ha señalado en la introducción de este artículo (Martínez-Tur, Peiró, Moliner y Potocnik, 2010; Schalock, 2015; Schalock y Verdugo, 2007, 2013a, 2013b).

También se alimenta, como también se ha indicado, de las propuestas planteadas dentro del modelo de impacto colectivo (Kania y Kramer, 2011). Facilitar las condiciones para que se logre un impacto colectivo y transformador requiere de un plan concreto que, en nuestro caso, contempla las siguientes fases y acciones (Figura 3):

1. En primer lugar, todos los participantes tienen que tener una visión compartida para la transformación, tienen una comprensión común del problema y se esfuerzan en común desarrollando acciones para avanzar. Esto implica tener un buen conocimiento del modelo que implica el cambio de paradigma, un liderazgo comprometido y transformacional, e implica que actúe FEAPS como anfitrión y coordinador.

2. En segundo lugar, se solicita desde la voluntariedad la aceptación de realizar el viaje, se pide formalmente que haya un compromiso reformador de los responsables de la organización y se pide que se configure un equipo promotor transversal (en el que, de forma obligatoria, haya al menos una persona con discapacidad intelectual o del desarrollo -por ejemplo, en atención temprana esto 
no aplicaría por razones obvias-, un familiar, un profesional, un responsable del servicio y, en su caso, un voluntario).

3. En tercer lugar, se promueve que haya unas medidas comunes (extraídas de herramientas relevantes para el proyecto, en nuestro caso especialmente centradas en la evaluación del impacto de los servicios en la calidad de vida de las personas), una comunicación constante entre los equipos comprometidos, a través de nuestros grupos colaborativos en la Intranet, y un plan de trabajo realista, con la colaboración y coordinación de FEAPS. Esto implica:

- contar con datos que nos den una foto de la realidad de un servicio antes del viaje,

- comparar la foto con la del modelo y con la de los demás viajeros,

- ofrecer oportunidades de formación (en relación con el modelo deseado), asesoramiento y acompañamiento,

- implica también que cada servicio diseñe y lleve a cabo un proyecto positivo y posible, sencillo (microtransformaciones), que sea singular, explícito y compartido,

- promover la interacción entre los compañeros de viaje y la innovación y, finalmente,

- mostrar el viaje realizado a todos los integrantes de proyectos y a otros que deseen emprender ese camino, de modo que se reconozca y se celebre el valor de su aventura y así trasladar el anhelo del viaje a otros.

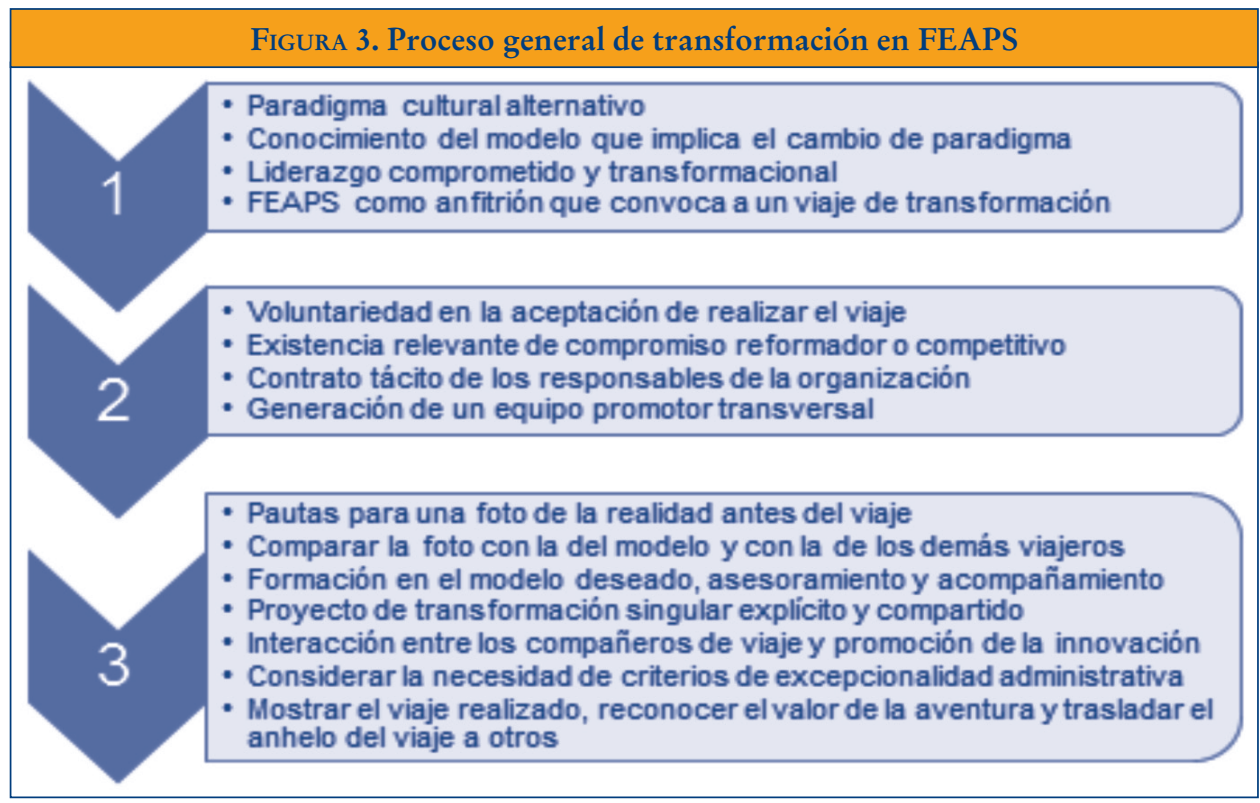

(C) Ediciones Universidad de Salamanca

Siglo Cero, vol. 46 (3), n. ${ }^{\circ} 255,2015$, julio-septiembre, pp. 47-71

$$
-68-
$$


En el conocimiento sobre los procesos de transformación organizacional se hace hincapié, como ya se ha resaltado, en la importancia del cambio cultural como clave para una verdadera y profunda transformación (Martínez-Tur, Peiró, Moliner y Potocnik, 2010). En FEAPS, estamos en un proceso de cambio cultural desde 1996 para orientar nuestras políticas, nuestras prácticas y nuestros servicios a la calidad de vida y los derechos de las personas con discapacidad intelectual o del desarrollo.

En el caso de FEAPS entendemos, por tanto, que el modelo de calidad de vida (Schalock y Verdugo, 2003) debe estar en la base de todo proceso de transformación y se debe contar con las propias personas con discapacidad para desarrollarlo (Schalock y Verdugo, 2007, 2013a y 2013b; Mansell, Knapp, Beadle-Brown y Beecham, 2007). Por lo tanto, en coherencia con nuestra misión, en cumplimiento de los derechos de las personas con discapacidad y reconociendo la realidad de nuestro entorno, necesitamos llevar a cabo proyectos piloto de apoyo a prácticas de transformación organizacional para mejorar la calidad de vida y lograr el ejercicio de los derechos de las personas con discapacidad intelectual y que inspiren a otras organizaciones a seguir ese camino. Queremos y necesitamos generar visión y pasión por la transformación, y queremos y necesitamos hacerlo en la práctica, de la mano de proyectos que se basen en información contrastada y datos relevantes, basados en el conocimiento científico más actualizado y en metodologías de transformación, que se gesten desde la base de la ciencia y los derechos, y contando con la participación de las propias personas con discapacidad intelectual o del desarrollo, sus familiares, los profesionales y gestores de los servicios. Los 104 servicios actualmente en el proyecto de transformación de FEAPS son una buena base de partida y una evidencia del compromiso asumido.

\section{Referencias bibliográficas}

Agora [agoranews] (2014, octubre 14). Transformemos el horizonte: hacia servicios de apoyo centrados en la persona [Archivo de video]. Recuperado el 31 de marzo de 2015 de https:// www.youtube.com/watch?v=XoOJHnBkvuU.

Amado, A. N. y McBride, M. (2001). Increasing Person-Centered Thinking: Improving the Quality of Person-Centered Planning: A Manual for Person-Centered Planning Facilitators. Minneapolis, Minnesota: University of Minnesota, Institute on Community Integration.

Bason, C. (Dir.) (2013). Powering European Public Sector Innovation: Towards A New Architecture. Report of the Expert Group on Public Sector Innovation. Bruselas: Comisión Europea.

Boletín Oficial del Estado (2008). BOE 96, 21 de abril de 2008, 20648-20659. Madrid: BOE.

Bradley, V. J. (1994). Evolution of a new service paradigm. En V. J. Bradley, J. W. Ashbaugh y B. C. Blaney (Eds.), Creating individual supports for people with developmental disabilities: A mandate for change at many levels (pp. 11-32). Baltimore: Paul H. Brookes.

Comisión Europea (2013). Unión por la Innovación. Una guía de bolsillo sobre una iniciativa de Europa 2020. Luxemburgo: Oficina de Publicaciones de la Unión Europea. Recuperado el 31 de marzo de 2015 de http://bookshop.europa.eu/en/innovation-union-pbKI3213062/ downloads/KI-32-13-062-ES-C/KI3213062ESC_002.pdf?FileName=KI3213062ESC_ 002.pdf\&SKU=KI3213062ESC_PDF\&CatalogueNumber=KI-32-13-062-ES-C. 
Dirección General de Empleo, Asuntos Sociales e Igualdad de Oportunidades (2011). Informe del grupo de expertos ad hoc sobre la transición de la asistencia institucional a la de base comunitaria (Bruselas, Comisión Europea, 2009). Zerbitzuan, 49, 147-160.

FEAPS (2009). La educación que queremos. Madrid: FEAPS.

FEAPS (2010). La misión de FEAPS. Madrid: FEAPS. Recuperado el 31 de marzo de 2015 de http://www.feaps.org/archivo/centro-documental/doc_download/305-.html.

FEAPS (2013). Declaración de Alcalá. Madrid: FEAPS. Recuperado el 31 de marzo de 2015 de http://www.feaps.org/files/Declaración\%20Diritentes\%20Alcalá_juli2013.pdf.

FEAPS CONFEDERACión [comunicacionfeaps] (2014, diciembre 15 y 16). Avances en la transformación de servicios de Atención Temprana [Archivo de video]. Recuperado el 31 de marzo de 2015 de https://www.youtube.com/watch?v=RWJMCTuIgjA; https://www.youtube. $\mathrm{com} /$ watch? $\mathrm{v}=\mathrm{onUX} 3 \mathrm{X} 4 \mathrm{Tgq} Y \mathrm{https} / /$ www.youtube.com/watch? $\mathrm{v}=\mathrm{rdz0iXb1008}$.

González, B., Ortega, E., Martínez, B. y Tamarit, J. (2014). Hacia el logro de una vida deseada: la experiencia de la Planificación Centrada en la Persona con personas con discapacidad intelectual o del desarrollo en España. En P. Rodríguez y A. Vilá (Coords.), Modelo de atención integral y centrada en la persona. Teoría y práctica en ámbitos del envejecimiento y la discapacidad (cap. 15). Madrid: Tecnos.

Grande, E., Hernández, S. y Tamarit, J. (2015). SECF: Sistema de Evaluación de Calidad FEAPS. Análisis de las acciones de mejora desarrolladas por las entidades acreditadas como base para el desarrollo de herramientas y apoyos. Siglo Cero, número extraordinario. IX Jornadas Científicas Internacionales de Investigación sobre Discapacidad.

Hickey, R. (2012). End-users, public services, and industrial relations: the restructuring of social services in Ontario. Industrial relations, 67, 4, 590-611.

Inclusion Internacional (C. Laurin-Bowie, Dir.) (2012). Comunidades Inclusivas = Comunidades más fuertes: Informe Mundial sobre el Artículo 19: El Derecho a Vivir y Ser Incluido en la Comunidad. Londres: Inclusion International.

InClusion International (C. LAURIN-Bowie, Dir.) (2014). Independiente pero no solo. Informe mundial sobre el derecho a decidir. Londres: Inclusion International.

Kania, J. y Kramer, M. (2011). Collective Impact. Stanford Social Innovation Review, Winter, 36-41.

LACASTA, J. J. (2015). FEAPS, 50 años de unión por las personas con discapacidad intelectual y por sus familias. Siglo Cero, 46 (1), 253, 41-65.

Mansell, J. y Beadle-Brown, J. (2011). Desinstitucionalización y vida en la comunidad: declaración del Grupo de Investigación sobre Política y Práctica Comparativas, de la Asociación Internacional para el Estudio Científico de las Discapacidades Intelectuales (IASSID). Zerbitzuan, 49, 137-146.

Mansell, J., Knapp, M., Beadle-Brown, J. y Beecham, J. (2007). Deinstitutionalisation and community living - outcomes and costs: report of a European Study. Volume 1: Executive Summary. Canterbury: Tizard Centre, University of Kent.

Martínez-Tur, V., Peiró, J. M., Moliner, C. y Potocnik, K. (2010). Calidad de servicio $y$ calidad de vida: el survey feedback como estrategia de cambio organizacional. Madrid: FEAPS.

Martínez-Tur, V. y Peñarroja, V. (2012). Innovación en el Tercer Sector ¿Crisis de Iniciativa? Madrid: ICONG.

Morgan, S. (2010). Opportunities and risks of personalisation. Nursing Management, 17, 2, 14-17.

Ministerio de Sanidad, Política Social e Igualdad (2011). Estrategia española sobre discapacidad 2012-2020. Madrid: Ministerio de Sanidad, Política Social e Igualdad. Recuperado 
el 31 de marzo de 2015 de http://www.lamoncloa.gob.es/espana/eh14/social/Documents/ estrategia_espanola_discapacidad_2012_2020.pdf.

Mount, B. (2000). Person-centered planning: Finding directions for change using personal futures planning. Amenia, NY: Capacity Works.

PARKer, C. (2011). A community for all Checklist. Implementing article 19 of de Convention on Rights of Persons with Disabilities. Open Society Public Health Program. New York: Open Society Foundations.

Schalock, R. L. (2015). Las mejores prácticas de las organizaciones y los profesionales. Siglo Cero, 46 (1), 253, 7-23.

Schalock, R. L., Borthwick-Duffy, S. A., Bradley, V. J., Buntinx, W. H. E., Coulter, D. L., Craig, E. M., Gomez, S. C., Lachapelle, Y., Luckasson, R., Reeve, A., Shogren, K. A., Snell, M. E., Spreat, S., Tassé, M. J., Thompson, J. R., Verdugo-Alonso, M. Á., Wehmeyer, M. L. y Yeager, M. H. (2010). Intellectual disability. Definition, classification, and systems of supports. 11th Edition. Washington, D. C.: American Association on Intellectual and Developmental Disabilities (AAIDD). Traducción española (2011): Discapacidad intelectual. Definición, clasificación y sistemas de apoyo. 11. ${ }^{a}$ edición. Madrid: Alianza.

Schalock, R. L., Gardner, J. y Bradley, V. (2007). Quality of Life for People with Intellectual and Other Developmental Disabilities: Applications across Individuals, Communities, and Systems. Washington: AAIDD. Traducción española (2008): Calidad de Vida para personas con discapacidad intelectual y otras discapacidades del desarrollo. Aplicaciones para personas, organizaciones, comunidades y sistemas. Madrid: FEAPS.

Schalock, R. L. y Verdugo, M. Á. (2003). Calidad de Vida: manual para profesionales de la educación, salud y Servicios Sociales. Madrid: Alianza Editorial.

Schalock, R. L. y Verdugo, M. Á. (2007). El concepto de Calidad de vida en los servicios y apoyos para personas con discapacidad intelectual. Siglo Cero, 224, 21-36.

Schalock, R. L. y Verdugo, M. Á. (2013a). El cambio en las organizaciones de discapacidad. Estrategias para superar sus retos y bacerlo realidad. Guía de liderazgo. Madrid: Alianza.

Schalock, R. L. y Verdugo, M. Á. (2013b). The transformation of disabilities organizations. Intellectual and Developmental Disabilities, 51, 4, 273-286.

TAMArit, J. (2006). Calidad FEAPS: un modelo para la calidad de vida, desde la ética y la calidad del servicio. En M. Á. Verdugo y B. Jordán de Urríes (Coords.), Rompiendo inercias, claves para avanzar (pp. 163-178). Salamanca: Amarú.

TAmarit, J. (2010). La calidad como presupuesto de los servicios de apoyo a las personas con discapacidad. En Luis Cayo Pérez Bueno (Dir.), Discapacidad, Tercer Sector e Inclusión Social. Estudios en Homenaje a Paulino Azúa Berra (pp. 309-328). Madrid: CERMI.

TAMARIT, J. (2012). Buenas prácticas FEAPS: en busca de prudencia y compromiso. En M. Á. Verdugo, T. Nieto, M. Crespo y B. Jordán (Coords.), Cambio organizacional y apoyo a las graves afectaciones. Dos prioridades (pp. 157-170). Salamanca: Amarú.

TAMARIT, J. (2014). La transformación necesaria de los centros y servicios: hacia sistemas de apoyos centrados en la persona. En III Congreso internacional de autismo. Murcia. Informe no publicado.

TAmarit, J. y Espejo, L. (2013). Experiencias de empoderamiento en personas con discapacidad intelectual o del desarrollo. Siglo Cero, 44, 2, 26-39.

Van Loon, J. (2006). Los apoyos en Arduin. Sobre las diversas facetas de los apoyos y la implementación de la Escala de Intensidad de Apoyos (SIS) en una organización de servicios. Siglo Cero, 37, 4 (220), 17-33.

Walker, P. (2012). Strategies for organizational change from group homes to individualized supports. Intellectual and Developmental Disabilities, 50, 5, 403-414. 